\title{
Poster Abstract: Robust Detection of Motor-Produced Audio Signals
}

\author{
Adeola Bannis \\ Carnegie Mellon University \\ Pittsburgh, Pennsylvania 15213 \\ abannis@andrew.cmu.edu
}

\author{
Hae Young Noh \\ Carnegie Mellon University \\ Pittsburgh, Pennsylvania 15213 \\ noh@cmu.edu
}

\author{
Pei Zhang \\ Carnegie Mellon University \\ Moffett Field, California 94035 \\ peizhang@cmu.edu
}

\begin{abstract}
Indoor localization systems cannot rely on the same mechanisms, like GPS, that are used for outdoor or large-scale localization. Instead, autonomous or user-carried devices are often localized by measuring the time taken for an emitted signal to reach a known location; this signal can be sound, light, radio waves, or another similar sensed quantity. $\mathrm{Au}-$ tonomous mobile devices already contain motors, which produce sounds as a side effect of their operation, and so can potentially be included in a localization scheme without new hardware. In this paper, we briefly outline the challenges that need to be met for accurate detection and identification of motor-produced signals. We present a method for improving signal resolution for linear chirps that improves cross-correlation based signal detection by up to $2.8 \mathrm{X}$.
\end{abstract}

\section{CCS CONCEPTS}

- Computer systems organization $\rightarrow$ Sensors and actuators;

\section{KEYWORDS}

chirplet filter, indoor localization, motor side-channel

\section{ACM Reference format:}

Adeola Bannis, Hae Young Noh, and Pei Zhang. 2018. Poster Abstract: Robust Detection of Motor-Produced Audio Signals. In Proceedings of The 16th ACM Conference on Embedded Networked Sensor Systems, Shenzhen, China, November 4-7, 2018 (SenSys '18), 2 pages. DOI: $10.1145 / 3274783.3275209$

\footnotetext{
Permission to make digital or hard copies of all or part of this work for personal or classroom use is granted without fee provided that copies are not made or distributed for profit or commercial advantage and that copies bear this notice and the full citation on the first page. Copyrights for components of this work owned by others than ACM must be honored. Abstracting with credit is permitted. To copy otherwise, or republish, to post on servers or to redistribute to lists, requires prior specific permission and/or a fee. Request permissions from permissions@acm.org.

SenSys '18, Shenzhen, China

(c) 2018 ACM. $978-1-4503-5952-8 / 18 / 11 \ldots \$ 15.00$

DOI: $10.1145 / 3274783.3275209$
}

\section{INTRODUCTION}

Some Internet-of-Things (IoT) devices need to move around indoors to complete their tasks, such as cleaning, search or surveillance, but cannot rely on localization technologies like GPS that could be used outdoors. Sound-based localization is a common choice for indoor systems [2] [4] [5], as it uses affordable and common sensors/actuators (i.e. speakers/buzzers and microphones), with ranges on the order of magnitude of typical indoor spaces. However, sound-based systems may suffer when the device itself is a source of highamplitude sounds, e.g. an autonomous vacuum cleaner. We propose taking advantage of these high-amplitude motor sounds to create signals for sound-based localization. In particular, we focus on the characteristics of sound produced by digital pulse-width modulation (PWM) controlled motors.

In this paper, we describe how the differences between motor-produced and speaker-produced sound can affect the accuracy of signal detection and identification. We present a method to improve signal detection of linear chirps produced as a side effect of motor operations. These linear chirps can be used as target signals in a sound-based localization system.

\section{MOTOR SOUND DISTORTION}

A major difference between motor-produced PEWs and digital audio is the general "shape" of the PEW in the timefrequency domain. Ideally, a linear chirp signal appears as an increasingly smooth curve on the time-frequency plane as the time and/or frequency resolution of the STFT increases. However, the sound signals from PWM driven motors quickly resolve as step-like shapes, no matter how good of a resolution we can achieve. More importantly, the duration of the steps may be different for each device. The PWM controller in each device is typically scheduled to update at fixed intervals determined by the manufacturer. In some cases, the steps may vary in length as the tasks may not run exactly on time. LiLT aims to support as wide a range of IoT devices as possible, and so must be robust to the non-ideal signal shape. In order to reduce errors in ToA estimation, we propose a two-pass method for locating the desired signal in a recorded signal.

The first pass of the method is a standard cross-correlation between the template linear chirp and the actual recorded 

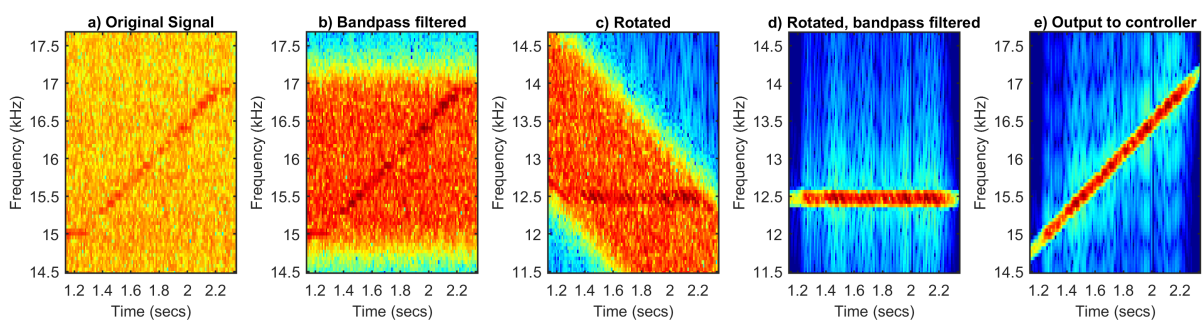

Figure 1: If a possible chirp is detected, the input is "chirp filtered" to extract a new template that will be crosscorrelated with the signal from other sensors.

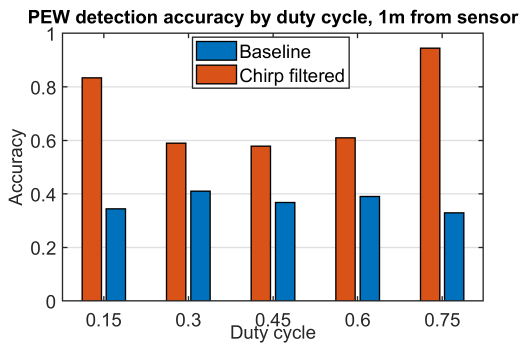

Figure 2: Adding a second chirp-filtering step improves detection accuracy up to $2.8 \mathrm{X}$.

signal. If the cross-correlation value is above a threshold, the second pass applies a "chirp filter" to the signal. This means applying a chirplet transform [3], applying a band-pass filter, then inverting the chirplet transform. This extracts the linear chirp-like part of the signal for use as a new template. This new template can be used to align the signals from other sensors, and thus to determine the true time of arrival of the motor-produced sound. The time-frequency content of a typical signal at each step is shown in Fig. 1.

\section{EVALUATION}

To evaluate the system, we used micro-quadcopters with small brushed DC motors [1] to generate the linear chirps. We set a single receiver in the centre of the room. One transmitter is placed at $1 \mathrm{~m}$ from the receiver, to represent an interfering motor that is not currently producing a signal. The duty cycle is set to $45 \%$, slightly higher than a typical hovering power for the Crazyflie. This reflects the expected operating behavior of a nearby quadcopter: in many sensing tasks it will not be accelerating significantly, but hovering in place to collect readings or update its position. The target transmitter is also set to $45 \%$ duty cycle, and the distance to the sensor was varied from 0.25 to $1.75 \mathrm{~m}$. Overall, the two-pass method improves the detection accuracy of motorproduced chirps, especially at extreme values of duty cycle.

\section{CONCLUSION}

Motor sounds produced by autonomous devices can be used in sound localization systems, as long as target signals can be distinguished from noise or normal operation. We have proposed a two-pass method to improve signal detection and localization of special motor-produced audio signals. Our method is nearly three times as accurate as cross-correlation template matching in the presence of interfering motor noise.

\section{REFERENCES}

[1] Bitcraze. 2017. CrazyFlie 2.0. https://www.bitcraze.io/crazyflie-2/. (2017).

[2] Patrick Lazik and Anthony Rowe. 2012. Indoor pseudo-ranging of mobile devices using ultrasonic chirps. In Proceedings of the 10th ACM Conference on Embedded Network Sensor Systems. ACM, 99-112.

[3] Steve Mann and Simon Haykin. 1995. The chirplet transform: Physical considerations. IEEE Transactions on Signal Processing 43, 11 (1995), 2745-2761.

[4] Zheng Sun, Aveek Purohit, Kaifei Chen, Shijia Pan, Trevor Pering, and Pei Zhang. 2011. PANDAA: physical arrangement detection of networked devices through ambient-sound awareness. In Proceedings of the 13th international conference on Ubiquitous computing. ACM, 425-434.

[5] Stephen P Tarzia, Peter A Dinda, Robert P Dick, and Gokhan Memik. 2011. Indoor localization without infrastructure using the acoustic background spectrum. In Proceedings of the 9th international conference on Mobile systems, applications, and services. ACM, 155-168. 